\title{
EPIDEMIOLOGIA DO TRAUMATISMO CRANIOENCEFÁLICO NO ESTADO DE PERNAMBUCO NO ANO DE 2017
}

\author{
Alyne Garcia Costa da Paixão ${ }^{1}$ \\ Rafaella Rayssa Ramos de Barros ${ }^{2}$ \\ Robert Gomes da Paixão ${ }^{3}$ \\ Andrea Vasconcelos de Santana ${ }^{4}$
}

\section{RESUMO}

Introdução: o traumatismo cranioencefálico (TCE) é um problema de saúde pública que acomete principalmente os jovens, gerando grande impacto nos cofres públicos. Os danos causados pelas lesões podem levar a vítima a óbito ou acarretar sequelas que impactam direta ou indiretamente a vida do paciente e da família. Objetivo: identificar o perfil epidemiológico das vítimas de TCE em Pernambuco com foco nas principais causas externas e características dos vitimados, como: idade, sexo e local de residência. Metodologia: trata-se de um estudo de caráter epidemiológico descritivo e transversal que será realizado por meio de dados públicos disponibilizado na plataforma de epidemiologia e morbidade do Departamento de Informática do Sistema Único de Saúde (DATASUS) e sendo inclusos pacientes atendidos no período de 2017 no Estado de Pernambuco, analisados de acordo com a idade, sexo, raça, macrorregião de saúde e causas externas. Resultados: A Macrorregião Metropolitana possui a maior prevalência dos casos de TCE, indivíduos na faixa etária de 20-44 anos, do sexo masculino e da cor/raça parda são os mais acometidos. Considerações finais: A partir das informações obtidas neste estudo será possível gerar atualização dos dados sobre TCE em Pernambuco, para que ações preventivas possam ser traçadas de acordo com o perfil dos acidentes.

Palavras-chave: Traumatismo Cranioencefálico. Epidemiologia. Morbidade. Mortalidade.

\footnotetext{
${ }^{1}$ Bacharela em Enfermagem.

${ }^{2}$ Bacharela em Enfermagem.

${ }^{3}$ Bacharel em Enfermagem.

${ }^{4}$ Bacharelanda em Fisioterapial.
} 


\title{
EPIDEMIOLOGY OF CRANIOENCEPHALIC TRAUMATISM IN THE STATE OF PERNAMBUCO IN THE YEAR 2017
}

\begin{abstract}
Introduction: traumatic brain injury (TBI) is a public health problem that mainly affects young people and generates high costs for public coffers. Damage caused by injuries can lead to death or sequelae that directly or indirectly impact the life of the patient and family. Objective: To identify the epidemiological profile of TBI victims in Pernambuco, focusing on the main external causes and characteristics of victims, such as: age, sex and place of residence. Methodology: this is a descriptive and cross-sectional epidemiological study that will be carried out using public data available on the epidemiology and morbidity platform of the Department of Informatics of the Unified Health System (DATASUS) and including patients seen in the period of 2017 in the State of Pernambuco, analyzed according to age, sex, race, macroregion of health and external causes. Results: The metropolita macroregion has the highest prevalence of TBI cases, individuals in the age group of 20-44 years, males and brown / color are the most affected. Final considerations: From the information obtained in this study it will be possible to update the data on TBI in Pernambuco,so that preventive actions can be traced according to the accident profile.
\end{abstract}

Keywords: Cranioencephalic trauma. Epidemiology. Morbidity. Mortality. 


\section{INTRODUÇÃO}

O traumatismo cranioencefálico (TCE) é originado em decorrência de qualquer agressão que venha a provocar quaisquer lesões no crânio e/ou encéfalo (MAGALHÃES et al., 2017). O TCE pode ser primário: quando o encéfalo é atingido diretamente junto com as estruturas vasculares, incluindo contusões, hemorragias, lacerações e outras lesões no cérebro e membranas, ou secundário: quando os agravos são decorrentes das lesões primárias. As complicações de maior gravidade são os picos muito elevados da pressão intracraniana (PIC) e a herniação cerebral (PHTLS 2017, p. 258-288).

Dessa forma, os fatores como idade, tipo de lesão, febre, gravidade do trauma, avaliação de pequenas sequelas, pressão arterial, baixa pontuação na Escala de Coma de Glasgow (ECG) e avaliação das sequelas interferem na evolução e recuperação do paciente, influenciando diretamente no prognóstico (OLIVEIRA et al,2017). As principais complicações ocasionadas pelo TCE podem ser evitadas com o diagnóstico prévio, através da tomografia computadorizada (TC) que também é utilizada para dimensionar e identificar a área afetada pelo trauma (GAUDÊNCIO E LEÃO, 2013).

Assim, cerca de 1,5 milhão de brasileiros são vítimas de TCE ocasionados por acidentes de trânsito (AT) e aproximadamente 100 mil evoluem para óbito (RODRIGUES et al. 2018). O aumento significativo da frota de motocicletas também está relacionado com a elevação do índice de TCE por acidentes moto ciclísticos em algumas regiões do Brasil. Outras causas envolvem lesões por armas de fogo e quedas (MAGALHÃES et al., 2017). Já incidência de causas nas mulheres se resume as quedas, agressões físicas e atropelamentos, diferente dos homens (RODRIGUES et al. 2018).

Por isso há uma relação entre a prevalência de TCE em homens com até 45 anos e os fatores comportamentais relacionados a idade, estando essa 
faixa etária mais exposta aos riscos de AT (MAGALHÃES et al., 2017). Para Passos et al. (2015), as principais causas são: acidentes de moto e as agressões físicas, sendo que, os acidentes ocorreram com mais frequência aos domingos e as agressões aos sábados. Entretanto, existem variáveis que não foram estudadas, devido à falta de preenchimento correto das informações nos prontuários. De acordo com Monteiro et al. (2016) existe ainda uma grande dificuldade em contabilizar o número de casos de TCE no Brasil, devido à ausência de registros e a dificuldade em identificá-lo em pacientes politraumatizados.

Consequentemente os impactos sofridos pelas vítimas de TCE são as sequelas físicas, evidenciadas em curto prazo como as alterações musculares, endócrinas, no trato gastrointestinal e urinário, como também problemas relacionados à dinâmica respiratória, além do comprometimento do sistema motor.

De médio a longo prazo o TCE pode ocasionar problemas de personalidade e comportamentais, como medo excessivo, insegurança, baixa autoestima, depressão e desinibição. Os pacientes com TCE são mais susceptíveis a déficits de atenção, concentração e orientação principalmente na fase aguda da lesão (BRASIL, 2017).

Assim sendo, cotidianamente o indivíduo pode apresentar agressividade, irritabilidade e estresse emocional, muitas vezes ocasionado pela dificuldade ou incapacidade de realizar tarefas simples que antes eram comuns na vida do paciente. A família é indispensável no processo de reabilitação e reinserção desse paciente, onde, o ambiente familiar muitas vezes é um meio facilitador da reabilitação, e é através dele que o paciente vai ser reintegrado no seu contexto familiar e sociocultural (BRASIL, 2017).

Nesse contexto, pode ser relevante identificar as características epidemiológicas do TCE, possibilitando o desenvolvimento de medidas preventivas para a redução desse agravo e suas sequelas nas vítimas 
(MONTEIRO et al., 2016). Dessa forma, o presente trabalho teve o objetivo de descrever a epidemiologia do TCE em Pernambuco no ano de 2017.

\section{METODOLOGIA}

O estudo realizado é de caráter epidemiológico, descritivo e transversal com abordagem quantitativa. A coleta de informações se deu no período de fevereiro a novembro de 2018 na base de dados fornecidos pelo Departamento de Informática do Sistema Único de Saúde (DATASUS) na plataforma de morbidade hospitalar e Sistema de Informações Hospitalares do SUS (SIH/SUS)

Critérios de inclusão: possuir residência no Estado de Pernambuco (PE), atendimentos realizados no ano de 2017 em PE com residência nas macrorregiões de saúde, sexos masculino, feminino e ignorado; na faixa etária que compreende menores de 1 e acima de 80 anos, pertencentes a cor/raça branca, preta, parda, amarela, indígena e CID-10 (traumatismo intracraniano) e grande grupo de causas (acidentes de transporte, outras causas externas de traumatismos acidentais e agressões). Critério de exclusão: os indivíduos cujos CID's foram incompatíveis com o TCE. Para a análise dos dados e formatação das tabelas foi utilizado o programa da Microsoft: Excel versão 2007. Tomando como referencial a resolução $\mathrm{n}^{\circ} 510$ de 7 de abril de 2016 do Conselho Nacional de Saúde o presente trabalho não necessita de aprovação do Comitê de Ética e Pesquisa, uma vez que, as informações foram obtidas através de dados públicos.

\section{RESULTADOS E DISCUSSÃO}

De acordo com o Quadro 1 a Região Metropolitana (RM) apresentou 69,06\% (34.163) dos internamentos e 70,79\% dos óbitos por TCE, dessa forma, sendo a região com maior prevalência. A região menos prevalência foi 0 Vale do São Francisco/Araripe com 7,44\% (3.863) dos internamentos e 4,83\% (66) dos óbitos. Internações por TCE em PE com 83,5\%, agressão foi a causa com menor percentual $2,74 \%$ de internamentos e $4,61 \%$ de óbitos. 


\section{Quadro 1- Internações e Óbitos por Traumatismo Cranioencefálico segundo as causas e as Macrorregião de Saúde em Pernambuco no ano de 2017}

\begin{tabular}{|c|c|c|c|c|c|c|c|c|}
\hline \multirow{2}{*}{$\begin{array}{l}\text { Macrorregião de } \\
\text { Saúde }\end{array}$} & $\begin{array}{l}\text { Acidentes de } \\
\text { transporte }\end{array}$ & $\begin{array}{l}\text { Outras causas } \\
\text { externas } \\
\text { acidentais }\end{array}$ & \multicolumn{2}{l|}{ Agressões } & \multicolumn{2}{l|}{ Total } \\
\cline { 2 - 10 } & $\begin{array}{c}\text { internamentos/ } \\
\text { obitos }\end{array}$ & $\begin{array}{l}\text { internamentos/ } \\
\text { Obitos }\end{array}$ & $\begin{array}{l}\text { internamentos/ } \\
\text { Obitos }\end{array}$ & \multicolumn{2}{|l|}{$\begin{array}{l}\text { internamentos/ } \\
\text { Óbitos }\end{array}$} \\
\hline Metropolitana & 4.620 & 40 & 28.669 & 875 & 874 & 52 & 34.163 & 967 \\
\hline Agreste & 502 & - & 6.873 & 227 & 12 & - & 7.387 & 227 \\
\hline $\begin{array}{c}\text { Sertão } \\
\text { Pernambucano }\end{array}$ & 141 & 11 & 4.085 & 95 & 13 & - & 4.239 & 106 \\
\hline $\begin{array}{c}\text { Vale São } \\
\text { Francisco/Araripe }\end{array}$ & 1.535 & 23 & 1.691 & 32 & 457 & 11 & 3.683 & 66 \\
\hline Total & $\mathbf{6 . 7 9 8}$ & $\mathbf{7 4}$ & $\mathbf{4 1 . 3 1 8}$ & $\mathbf{1 . 2 2 9}$ & $\mathbf{1 . 3 5 6}$ & $\mathbf{6 3}$ & $\mathbf{4 9 . 4 7 2}$ & $\mathbf{1 . 3 6 6}$ \\
\hline
\end{tabular}

Fonte: Ministério da Saúde - Sistema de Informações Hospitalares do SUS (SIH/SUS)

Neste estudo foi visto que a maior causa de internamentos por TCE em PE no ano de 2017 foi de $83,52 \%$, onde podemos destacar as quedas, a exposição a forças animadas e inanimadas, exposição a fatores não especificados, dentre outros.

Os acidentes de transporte, mais especificamente os acidentes moto ciclísticos, são os principais fatores de internações por TCE (MAGALHÃES et al., 2017). A nível de Nordeste, Moura et al. (2011) relata em seu estudo que em Petrolina, município localizado na macrorregião Vale São Francisco- PE, a maior prevalência de causa para o TCE foram os acidentes moto ciclísticos $(44,55 \%)$, porém as quedas e os atropelamentos também tiveram notoriedade, possuindo respectivamente $22,78 \%$ e $11,88 \%$ do total de casos, seguido das agressões $(5,94 \%)$, corroborando com este estudo onde indicou que as agressões em PE tiveram a menor prevalência de TCE.

Assim, relacionando o total de óbitos com o total de internamentos de cada causa, as agressões possuem o maior percentual (4,65\%), apesar de representar o menor número absoluto, nos mostrando que a violência urbana é um grande colaborador nas causas de óbitos por trauma cranioencefálico. Os 


\section{Revista FLAMMAE}

Revista Científica do Corpo de Bombeiros Militar de Pernambuco

Artigo Publicado no Vol.07 N.18 - Edição Jan a Jun 2021 - ISSN 2359-4829

Versão on-line disponível em: http://www.revistaflammae.com

motivos destas agressões devem receber um olhar mais detalhado, em prol de formas preventivas para atenuar os números desse agravo.

Em 2017, ocorreram 49.472 internamentos por TCE, onde a faixa etária mais prevalente foi entre 20 e 44 anos $(29,20 \%)$. A menos acometida foram os menores de 1 ano $(0,58 \%)$. Entre as regiões, a Metropolitana apresentou $69,06 \%$ dos vitimados, sendo a macrorregião com maior número de internamentos e a de menor número, o Vale São Francisco/Araripe com 7,44\%, conforme o quadro abaixo.

\section{Quadro 2- Internações por Traumatismo Cranioencefálico segundo Faixa Etária e Macrorregião em Pernambuco no ano de 2017}

\begin{tabular}{|c|c|c|c|c|c|}
\hline Faixa Etária 2 & Metropolitana & Agreste & $\begin{array}{c}\text { Sertão } \\
\text { Pernambucano }\end{array}$ & $\begin{array}{c}\text { Vale São } \\
\text { Francisco/ } \\
\text { Araripe }\end{array}$ & Total \\
\hline Menor 1 ano & 203 & 43 & 23 & 18 & 287 \\
\hline 1 a 4 anos & 1.366 & 291 & 144 & 94 & 1.895 \\
\hline 5 a 9 anos & 1.447 & 355 & 216 & 190 & 2.208 \\
\hline 10 a 14 anos & 1.811 & 388 & 284 & 195 & 2.678 \\
\hline 15 a 19 anos & 2.352 & 668 & 340 & 332 & 3.692 \\
\hline 20 a 24 anos & 3.277 & 829 & 396 & 403 & 4.905 \\
\hline 25 a 29 anos & 2.941 & 745 & 341 & 396 & 4.423 \\
\hline 30 a 34 anos & 2.878 & 637 & 338 & 319 & 4.172 \\
\hline 35 a 39 anos & 2.817 & 604 & 337 & 360 & 4.118 \\
\hline 40 a 44 anos & 2.533 & 545 & 267 & 280 & 3.625 \\
\hline 45 a 49 anos & 2.213 & 413 & 207 & 220 & 3.053 \\
\hline 50 a 54 anos & 2.081 & 347 & 240 & 188 & 2.856 \\
\hline 55 a 59 anos & 1.831 & 307 & 180 & 132 & 2.450 \\
\hline 60 a 64 anos & 1.588 & 240 & 168 & 118 & 2.114 \\
\hline 65 a 69 anos & 1.309 & 194 & 164 & 98 & 1.765 \\
\hline 70 a 74 anos & 1.053 & 212 & 163 & 102 & 1.530 \\
\hline 75 a 79 anos & 912 & 229 & 118 & 71 & 1.330 \\
\hline $\begin{array}{l}80 \text { anos e } \\
\text { mais }\end{array}$ & 1.551 & 340 & 313 & 167 & 2.371 \\
\hline Total & 34.163 & 7.387 & 4.239 & 3.683 & 49.472 \\
\hline
\end{tabular}

Fonte: Ministério da Saúde - Sistema de Informações Hospitalares do SUS (SIH/SUS).

A faixa etária mais acometida foi a de pessoas jovens (20-44 anos) demostrando que estes estão mais expostos aos fatores de risco. Segundo 
Silva (2018) no Centro de reabilitação de indivíduos vitimados por TCE no Piauí, a faixa etária entre 18 e 59 anos $(81,68 \%)$ foi a mais acometida. Em Alagoas a idade entre 21 e 30 anos tiveram $(21,9 \%)$ do total da amostra, tornando-se a faixa etária com maior prevalência de TCE no Estado (NASCIMENTO, MACIEL E OLIVEIRA, 2017). Dessa forma, esta pesquisa aponta que, fatores comportamentais do indivíduo, como a inexperiência, a impulsividade e irresponsabilidade, influenciam nos casos de TCE (NASCIMENTO, MACIEL E OLIVEIRA, 2017).

Segundo este estudo, nas crianças residentes em PE, O TCE foi prevalente na macrorregião Metropolitana. Isso é relatado no estudo de Amorim et al. (2017) onde a RM corresponde ao maior número de crianças acometidas por TCE. A presente pesquisa mostra que a faixa etária com maior prevalência entre as crianças foi a de $5-9$ anos de idade $(4,47 \%)$, já para Amorim, a prevalência foram as crianças no primeiro ano de vida.

Há uma predominância de $78,25 \%$ de indivíduos da cor/raça Parda nas internações por TCE em PE no ano de 2017.

\section{Quadro 3- Internações de Cor/Raça por Macrorregiões em Pernambuco no ano 2017}

\begin{tabular}{|l|r|r|r|r|r|}
\hline \multicolumn{1}{|c|}{ Cor/raça } & Metropolitana & Agreste & $\begin{array}{c}\text { Sertão } \\
\text { Pernambucano }\end{array}$ & $\begin{array}{c}\text { Vale São } \\
\text { Francisco/ } \\
\text { Araripe }\end{array}$ & \multicolumn{1}{c|}{ Total } \\
\hline Branca & 551 & 307 & 81 & 122 & 1.061 \\
\hline Preta & 257 & 47 & 25 & 35 & 364 \\
\hline Parda & 27.833 & 5.980 & 3.428 & 1.471 & 38.712 \\
\hline Amarela & 507 & 245 & 84 & 163 & 999 \\
\hline Indígena & 4 & 2 & 5 & 5 & 16 \\
\hline & & & & & \\
S/ informação & 5.011 & 806 & 616 & 1.887 & 8.320 \\
\hline Total & $\mathbf{3 4 . 1 6 3}$ & $\mathbf{7 . 3 8 7}$ & $\mathbf{4 . 2 3 9}$ & $\mathbf{3 . 6 8 3}$ & $\mathbf{4 9 . 4 7 2}$ \\
\hline
\end{tabular}

Fonte: Ministério da Saúde - Sistema de Informações Hospitalares do SUS (SIH/SUS) 
Dessa forma, houve um número expressivo na categoria parda, sendo predominante em todas as regiões. Em um estudo realizado por Silva (2018), $52,27 \%$ das vítimas se auto declararam pardas. Segundo dados do IBGE (2017), a maior parte da população residente no Estado de PE se declara parda ou branca, sendo a primeira, dominante.

Segundo o quadro 4 as internações ocorreram em maior quantidade na macrorregião Metropolitana (69,06\%), e o sexo mais acometido em todas as macrorregiões foi o masculino $(69,73 \%)$.

Quadro 4- Internações por Sexo e Macrorregiões em Pernambuco no ano de 2017

\begin{tabular}{|l|r|r|r|}
\hline $\begin{array}{l}\text { Macrorregião } \\
\text { de Saúde }\end{array}$ & \multicolumn{1}{c|}{ Masculino } & \multicolumn{1}{c|}{ Feminino } & \multicolumn{1}{c|}{ Total } \\
\hline Metropolitana & 23.476 & 10.687 & 34.163 \\
\hline Agreste & 5.468 & 1.919 & 7.387 \\
\hline $\begin{array}{l}\text { Sertão de } \\
\text { Pernambuco }\end{array}$ & 2.829 & 1.410 & 4.239 \\
\hline $\begin{array}{l}\text { Vale São } \\
\text { Francisco }\end{array}$ & 2.725 & 958 & 3.683 \\
\hline Total & $\mathbf{3 4 . 4 9 8}$ & $\mathbf{1 4 . 9 7 4}$ & $\mathbf{4 9 . 4 7 2}$ \\
\hline
\end{tabular}

Fonte: Ministério da Saúde - Sistema de Informações Hospitalares do SUS (SIH/SUS)

O presente estudo estima que no ano de 2017, em Pernambuco, houve prevalência de casos de TCE na região Metropolitana (69,06\%). Quanto ao sexo dos vitimados, o sexo masculino $(69,73 \%)$ teve grande predominância dentro da amostra, isso ocorre devido ao fato dos homens estarem mais expostos aos riscos de acidentes de trânsito e a outros fatores comportamentais que eventualmente podem resultar em lesões por Traumatismo Cranioencefálico. No estudo de Barros, Furtado E Bonfim, 2015, realizado no Hospital da Restauração em Recife-PE, foi observado que 84,05\% dos vitimados por TCE eram do sexo masculino.

Entende-se que, mesmo em um espaço cronológico de dois anos entre esse estudo e esta pesquisa, o sexo masculino manteve-se como o mais acometido por TCE em PE, independente da causa e/ou região dentro do estado. O TCE acometeu principalmente os homens adultos numa faixa etária 
de 20 a 44 anos, vítimas de acidentes. A maioria dos óbitos foram provenientes das agressões (RODRIGUES et al. 2018).

\section{CONSIDERAÇÕES FINAIS}

A partir de tais informações vê-se uma necessidade de criar um programa de prevenção mais efetivo para essa faixa etária.

A pesquisa sobre a epidemiologia do TCE em PE revelou-se importante, visto que, há uma escassez de artigos sobre o assunto e os estudos disponíveis são antigos. A partir das informações obtidas neste estudo será possível a atualização dos dados sobre TCE em PE, para que assim ações preventivas possam ser traçadas de acordo com o perfil epidemiológico dos acidentes.

\section{REFERÊNCIAS}

Amorim et al. Perfil epidemiológico de crianças vítimas de trauma cranioencefálico. Revista de enfermagem UFPE online, Recife, 11(Supl. 10):4150-6, out., 2017. https://periodicos.ufpe.br/revistas/revistaenfermagem/article/view/231177/25151

Brasil, Ministério da Saúde. Secretaria de Atenção à Saúde. Diretrizes de atenção à reabilitação da pessoa com traumatismo cranioencefálico. Departamento de Ações Programáticas Estratégicas. 1ed, 27-36pg, BrasíliaDF, 2015.

http://bvsms.saude.gov.br/bvs/publicacoes/diretrizes atencao reabilitacao pes soa traumatisco cranioencefalico.pdf

Barros, Furtado E Bonfim. Características clínicas e epidemiológicas de motociclistas com trauma cranioencefálico atendidos em hospital de referência. Revista de enfermagem UERJ, Rio de Janeiro, 2015 jul/ago; 23(4):540-7. http://www.facenf.uerj.br/v23n4/v23n4a17.pdf

Dantas, Oliveira E Neto. Epidemiologia do traumatismo crânio encefálico (TCE) no Nordeste no ano de 2012. REBES (Pombal - PB, Brasil), v. 4, n. 1 p. 18-23, jan-mar, 2014.

http://www.gvaa.com.br/revista/index.php/REBES/article/view/2573/1985 
Revista Científica do Corpo de Bombeiros Militar de Pernambuco

Artigo Publicado no Vol.07 N.18 - Edição Jan a Jun 2021 - ISSN 2359-4829

Versão on-line disponível em: http://www.revistaflammae.com

Gaudêncio TG, Leão GM. A Epidemiologia do Traumatismo CrânioEncefálico: Um Levantamento Bibliográfico no Brasil. Revista de Neurociência 2013;21:814-8

http://www.revistaneurociencias.com.br/edicoes/2013/RN2103/revisao/814revis ao.pdf

Histórico/Apresentação. Disponível em:

$<$ http://datasus.saude.gov.br/datasus >. Acesso em: 10 abril 2018.

IBGE, Brasil, Censo Demográfico. Disponível em:

$<$ https://sidra.ibge.gov.br/tabela/3175\#resultado $>$. Acesso em: 05 dezembro 2018.

IBGE, Brasil, Pernambuco. Disponível em:

$<$ https://cidades.ibge.gov.br/brasil/pe/panorama $>$. Acesso em: 10 abril 2018.

Jerônimo, et al. Fatores relacionados ao prognóstico de vítimas de traumatismo cranioencefálico: uma revisão bibliográfica. Arquivo Brasileiro Neurocirurgia 33(3): 165-9, 2014.

http://files.bvs.br/upload/S/0103-5355/2014/v33n3/756167.pdf

Magalhães et al. Epidemiologia do Traumatismo Cranioencefálico no Brasil. Revista Brasileira de Neurologia 53(2):15-22, 2017.

WWW.REVISTANEUROCIENCIAS.COM.BR/EDICOES/2013/RN2103/REVISA O/814REVISAO.PDF

Maia, Bernardo Guimarães; Paula, Fábio Ribeiro Pedroso de; Cotta, Gabriela Drummond. Perfil Clínico-Epidemiológico das Ocorrências de

Traumatismo Cranioencefálico. Revista de Neurociência 2013; 21:786-10p http://www.revistaneurociencias.com.br/edicoes/2013/RN2101/original2101/786 original.pdf

Monteiro LF et al. Caracterização dos pacientes com traumatismo cranioencefálico. Arquivo Catarinense de Medicina 2016 jul-set; 45(3):02-16 http://www.acm.org.br/acm/seer/index.php/arquivos/article/view/107

Moura et al. Perfil clínico-epidemiológico de traumatismo cranioencefálico do hospital de urgências e traumas no munícipio de Petrolina, estado de Pernambuco. Arquivo Brasileiro de Neurocirurgia B30(3):99-104, 2011. http://files.bvs.br/upload/S/0103-5355/2011/v30n3/a2709.pdf

Nascimento, Maciel E Oliveira. Análise epidemiológica das internações por trauma cranioencefálico em um hospital de urgência e emergência.

Revista de enfermagem UFPE online, Recife, 11(Supl. 7):2864-70, jul., 2017. https://periodicos.ufpe.br/revistas/revistaenfermagem/article/view/10172/19191 
Passos et al. Perfil clínico e sociodemográfico de vítimas de traumatismo cranioencefálico atendidas na área vermelha da emergência de um hospital de referência em trauma em Sergipe. Arquivos Brasileiros de Neurocirurgia Vol. 34 No. 4/2015 https://www.thieme-connect.com/products/ejournals/abstract/10.1055/s-0035$\underline{1564886}$

PHTLS, Atendimento Pré-hospitalar Ao Traumatizado, 20127 Ed cap.9 217244

Silva. Análise das características dos indivíduos com sequelas de traumatismo cranioencefálico (TCE) em um centro de referência em reabilitação (características de tce). Revista Brasileira de Neurologia. 54(2):28-33, 2018. http://docs.bvsalud.org/biblioref/2018/07/907023/revista542v4-artigo4.pdf

Viégas et al. Traumatismo cranioencefálico em um hospital de referência no estado do Pará, Brasil: prevalência das vítimas quanto a gênero, faixa etária, mecanismos de trauma, e óbito. Arquivo Brasileiro de Neurocirurgia 32(1): 15-8, 2013.

http://files.bvs.br/upload/S/0103-5355/2013/v32n1/a3620.pdf 